vs text, and open (anyone can access) vs closed models (provider controls access via e-mail invitation or access code).

Methods We conducted five focus groups with potential users (youth, men who have sex with men (MSM), STI clinic clients), community agencies, and public health nurses, and interviewed family physicians, recruited through multiple methods. Participants were shown visual depictions of OPN models and examples of existing services, and opinions elicited using discussion guides that probed acceptability, advantages, and challenges of differing models. Notes taken were supplemented by review of audio recordings and analysed thematically.

Results We spoke with 16 potential users (6 youth, 6 MSM, 4 clients), 4 agency staff, 11 nurses, and 8 physicians. Older and younger users preferred OPN through e-mail and texting respectively, each perceiving the chosen modality as more serious and private. Participant points of convergence included: OPN are beneficial; need for two-stage messaging (initial generic, followed by detailed disease and contact information); few concerns regarding misuse; limitations given online sex-seeking without contact information. Most users preferred OPN for all possible STI while providers more commonly emphasised reportable or treatable infections. Users preferred closed access models which were perceived as more serious and secure. Providers preferred open models, perceiving closed models to create barriers for clients and difficult to integrate into clinical practice.

Conclusion We found overall support for OPN, but key differences between client and provider perceptions may pose challenges to uptake. As OPN are best promoted by providers giving an STI diagnosis, understanding and addressing provider concerns is important.

Disclosure of interest statement The authors have no conflicts of interest to disclose.

\section{P04.02 PARTNER NOTIFICATION IN NORTH AMERICA: A HISTORICAL ACCOUNT}

${ }^{1} \mathrm{O}$ Sobanjo, ${ }^{2} \mathrm{M}$ Steben*, ${ }^{*} \mathrm{E}$ Cheuk, ${ }^{4} \mathrm{~J}$ Kettner, ${ }^{3} \mathrm{M}$ Fast, ${ }^{4} \mathrm{H}$ Meadwick. ${ }^{1}$ Resident, Public Health and Preventive Medicine, Faculty of Medicine, McGill University, Montréal, Québec, Canada; ${ }^{2}$ STI Unit, Institut National de Santé Publique Du Québec, Montréal, Québec, Canada; ${ }^{3}$ Formerly From National Collaborative Centre on Infectious Diseases (NCCID), Winnipeg, Manitoba, Canada; ${ }^{4}$ National Collaborative Centre on Infectious Diseases, Winnipeg, Manitoba, Canada

10.1136/sextrans-2015-052270.256

Introduction Partner notification (PN) has been an integral part of sexually transmitted infection (STI) prevention for decades. Initially proposed for syphilis control, it now extends to a wide range of STI. NCCID PN project included documenting its origins in North America (NA).

Methods A multi-pronged approach has been adopted due to paucity of historical evidence.

- Literature search: electronic database (PubMed), manual reference list search, printed literature on public health $(\mathrm{PH})$ history in NA, search of significant $\mathrm{PH}$ organisation websites for related publications.

- Semi-structured interviews of key participants in STD control programs National STD prevention conference in Minnesota.

- Blogging: obtain historical accounts through use of online discussion sites or focus groups e.g. stdpreventiononline.org

- Request of materials: participants in the online blog and those for the structured interviews were to bring memorabilia, documents, letters.
Results PN might have been in use since the 19th century, PN has been shown effective when implemented as part of a larger $\mathrm{PH}$ initiative. Crucial to its success was the monitoring of performance. Confidentiality was a priority. Identifiers were used in reports to make it possible to trend networks and patterns. PN evolved in time with changes in diseases. Efforts were made to make available free services for testing and treatment. The costs implications favoured adoption of methods such as providerreferral and patient-referral. Given the importance of PN to STI prevention, it was disappointing to find limited documentation published particularly in Canada compared to United States.

Conclusion PN has been touted as the cornerstone in STI control. Looking at its origins and principles of success helps informs decisions regarding its current role and practice. Prevailing circumstances such as restricted budgets, high rates of mobility and increasing numbers of anonymous partners call for a modification in the use of PN today. Newer models employing social media are being developed.

Disclosure of interest statement The National Collaborating Centre for Infectious Diseases is funded by the Public Health Agency of Canada. No pharmaceutical grants were received in the development of this study.

\section{P04.03 WHICH PARTNER NOTIFICATION METHOD DO PATIENTS PREFER? RESULTS OF A PATIENT PREFERENCE SURVEY AT THE NATIONAL STI CLINIC IN SINGAPORE}

WS Tan*, TW Chio. Department of STI Control Clinic, Singapore

\subsection{6/sextrans-2015-052270.257}

Introduction Partner Notification (PN) can be made more effective if source patients are receptive to the method of PN. With increased mobile and internet connectivity, we carried out a survey to find out if our patient's preference towards PN had changed.

Methods A self-administered survey was carried out on clinic attendees over 4 weeks in February 2014. Respondents were asked about their preference for each PN method on a modified 4 point Likert scale.

Results A total of 416 completed survey forms were collected. The demographics of the respondents were consistent with the average clinic demographic: $74 \%(n=307)$ were male, $26 \%(n$ $=109$ ) were female. $71.6 \%$ were heterosexual, $23.6 \%$ were homosexual, and $4.8 \%$ bisexual. $90.5 \%$ had access to an Iphone/ Android or both.

If diagnosed with an STI, respondents rated the following methods as good/very good: Self notification (84.3\%), Phone call by clinic $(52.4 \%)$, SMS from clinic $(46.4 \%)$, Email sent by clinic (29.6\%), Letter sent by clinic (22.3\%).

If their partners had an STI, respondents rather the following PN methods as good/very good: Self notification (88.7\%), Phone call by clinic (54.1\%), SMS from clinic $(48.8 \%)$, Email sent by clinic (29.5\%), Letter sent by clinic (21.9\%).

Subgroup analyses found that significantly more respondents aged below 32 years preferred SMS $(52.6 \%$ vs. $42.2 \%, \mathrm{p}=$ 0.039 ). MSM respondents significantly preferred email as a PN method compared to heterosexual respondents $(41.5 \%$ vs. $26.5 \%, \mathrm{p}=0.03)$.

Conclusion Despite the advent of mobile connectivity, self-notification remains the most popular form of $\mathrm{PN}$ in our clinic attendees similar to the previous studies conducted in the United Kingdom. Respondents were also receptive to SMS PN, 
especially in younger age groups. This information would allow us to better allocate resources (e.g. provide an SMS messaging platform) and tailor PN methods according to age groups, sexual preference.

Disclosure of interest statement The authors report no conflict of interest in this study. The department of STI Control Clinic is funded by Ministry of Health, Singapore. No pharmaceutical grants were received in the development of this study.

\section{P04.04 CHLAMYDIA INFECTION IN MALES AND FEMALES REPORTING SEX WITH PARTNERS WITH CHLAMYDIA}

1,2,3,4S Huffam*, ${ }^{1,5}$ EPF Chow, ${ }^{1,5} \mathrm{CK}$ Fairley, ${ }^{2}$ J Hocking, ${ }^{1} \mathrm{~J}$ Peel, ${ }^{1,5} \mathrm{M}$ Chen. ${ }^{1}$ Melbourne Sexual Health Centre, Alfred Health, Melbourne, Victoria, Australia; ${ }^{2}$ School of Population and Global Health, University of Melbourne, Victoria, Australia; ${ }^{3}$ Department of Infectious Diseases, Barwon Health, Victoria, Australia; ${ }^{4}$ Royal Darwin Hospital, Northern Territory, Australia; ${ }^{5}$ Central Clinical School, Faculty of Medicine, Nursing and Health Sciences, Monash University, Victoria, Australia

\subsection{6/sextrans-2015-052270.258}

Background Justification for presumptive treatment for a sexually transmitted infection depends in part on the underlying prevalence of that infection among those reporting exposure. To examine these data for chlamydia we aimed to ascertain the proportion infected with chlamydia, and factors predictive of infection, amongst females, heterosexual males and men who have sex with men (MSM) presenting to a sexual health service reporting sexual contact with a chlamydia infected partner.

Methods Patients included were those attending the Melbourne Sexual Health Centre from October 2010 to September 2013. Proportions testing positive amongst females, heterosexual males, and MSM reporting sexual contact with a chlamydia infected partner were ascertained. Demographic and behavioural data obtained using computer assisted self-interview were analysed to determine predictive factors.

Results Of the 491 female, 808 heterosexual male, and 268 MSM chlamydia contacts, the proportion diagnosed with chlamydia were $39.9 \%$ (95\% CI 35.7-44.3), 36.1\% (95\% CI 32.939.9 ), and $23.5 \%$ (95\% CI 18.8-29.0) respectively. Female chlamydia contacts were more likely to have chlamydia if age $\leq 24$ (AOR 1.86, 95\% CI 1.12-3.10) or if they reported inconsistent condom use during vaginal sex with a regular male partner (AOR 2.5, 95\% CI 1.12-6.14). Heterosexual male contacts were more likely to have chlamydia if age $\leq 26$ (AOR $1.70,95 \%$ CI 1.26-2.30) or if they had a regular female sexual partner (AOR $1.42,95 \%$ CI 1.06-1.91). In MSM urethral chlamydia was diagnosed in $8.8 \%$, rectal chlamydia in $20.2 \%$, and in $3.9 \%$ at both sites. MSM were more likely to have chlamydia if they had a regular male sexual partner (OR 2.12 95\% CI 1.18-3.81).

Conclusion This study of female, heterosexual male and MSM presentations with self-reported chlamydia contact provides insight as to their likelihood of infection. The data may inform policy and individual clinical decision making regarding presumptive treatment of chlamydia contacts.

Disclosures No potential conflicts of interest.

\section{P04.05 HIGH PREVALENCE OF RECTAL GONORRHOEA AMONG MEN REPORTING CONTACT WITH MEN WITH GONORRHOEA: IMPLICATIONS FOR EPIDEMIOLOGICAL TREATMENT}

${ }^{1} \mathrm{~K}$ Dutt, ${ }^{1,2}$ EPF Chow*, ${ }^{*} \mathrm{~S}$ Huffam, ${ }^{1,3} \mathrm{~K}$ Klassen, ${ }^{1,2} \mathrm{CK}$ Fairley, ${ }^{1,2} \mathrm{CS}$ Bradshaw, ${ }^{1} \mathrm{I}$ Denham, ${ }^{1}$ MY Chen*. 'Melbourne Sexual Health Centre, Alfred Hospital, Melbourne, Victoria, Australia; ${ }^{2}$ Central Clinical School, Monash University, Melbourne, Victoria, Australia;

${ }^{3}$ Faculty of Medicine, Melbourne University, Melbourne, Victoria, Australia

10.1136/sextrans-2015-052270.259

Introduction This study aimed to determine the prevalence of gonorrhoea and factors associated with rectal gonorrhoea among men reporting sexual contact with men with gonorrhoea.

Methods Men who presented to Melbourne Sexual Health Centre reporting sexual contact with a male with gonorrhoea were prospectively identified between March 2011 and December 2013. These men were screened for pharyngeal and rectal gonorrhoea using culture. The prevalence of gonorrhoea among contacts was compared to that among all men who have sex with men (MSM) screened at the clinic over the same period. Logistic regression was performed on demographic and behavioural characteristics to determine the predictors of infection among contacts.

Results Among 363 contacts of gonorrhoea the prevalence of rectal gonorrhoea was 26.4\% (95\% CI: 21.8\%-31.0\%) compared to $3.9 \%$ (95\% CI: 3.7\%-4.2\%) among clinic attendees (p $<0.001$ ). The prevalence of pharyngeal gonorrhoea among contacts was 9.4\% (95\% CI: 6.4\%-12.4\%) compared to $2.1 \%$ (95\% CI: 1.9\%-2.4\%) among clinic attendees ( $p<0.001)$. Among contacts who reported not always using condoms during receptive anal sex with casual partners, rectal gonorrhoea was cultured in $42.4 \%$ compared with $12.7 \%$ among contacts reporting no receptive anal sex $(\mathrm{p}<0.001)$ and $20.2 \%$ among those reporting always using condoms $(\mathrm{p}<0.001)$. On multivariate analysis rectal gonorrhoea was associated with inconsistent condom use during receptive anal sex with casual partners (adjusted odds ratio (AOR): 4.16; 95\% CI: 1.87-9.26) and a reported past history of gonorrhoea (AOR: 1.77; 95\% CI: 1.01-3.14).

Conclusion The high proportion of positive cases of gonorrhoea among contacts in this study supports epidemiological treatment of MSM presenting as contacts of gonorrhoea.

Disclosure of interest There are no conflicts of interest to be disclosed relating to this paper.

\section{P04.06 PARTNER NUMBER AND OUTCOMES OF PARTNER NOTIFICATION AMONG GAY, BISEXUAL, AND OTHER MEN WHO HAVE SEX WITH MEN WITH INFECTIOUS SYPHILIS IN BRITISH COLUMBIA, CANADA}

${ }^{1,2} \mathrm{~J}$ Wong ${ }^{*},{ }^{1} \mathrm{M}$ Achen, ${ }^{1} \mathrm{~N}$ Holgerson, ${ }^{1,2} \mathrm{G}$ Ogilvie, ${ }^{1,2,3} \mathrm{M}$ Gilbert. ${ }^{1} B C$ Centre for Disease Control; ${ }^{2}$ University of British Columbia; ${ }^{3}$ Ontario HIV Treatment Network

\subsection{6/sextrans-2015-052270.260}

Background Since 2010, infectious syphilis (IS) cases in British Columbia (BC) have increased 4-fold among gay, bisexual, and 